\title{
3D-Printed Magnets Made from Recycled Materials
}

\section{Using recycled materials to make magnets could allow companies to circumvent supply chain issues for sourcing magnetic objects.}

\author{
By Katherine Wright
}

$\prod$ he COVID-19 pandemic threw a wrench in supply chains for many materials, including those used to make magnets. Magnets are crucial components in technologies ranging from computer hard-disk drives to magnetic resonance imaging scanners. To circumvent such disruptions, researchers are looking into alternative ways to source magnetic materials. Daniel Casaleiz of IMDEA Nanoscience, Spain, shared the details of one such method at the recent 2022 Joint Magnetism and Magnetic Materials (MMM) - Intermag conference, which took place online and in New Orleans in January.

Casaleiz and his colleagues have developed a method to recycle ferrite residues left over from the manufacture of commercial ferrite magnets, the world's most commonly produced type of magnet. First, the team collects strontium ferrite waste material

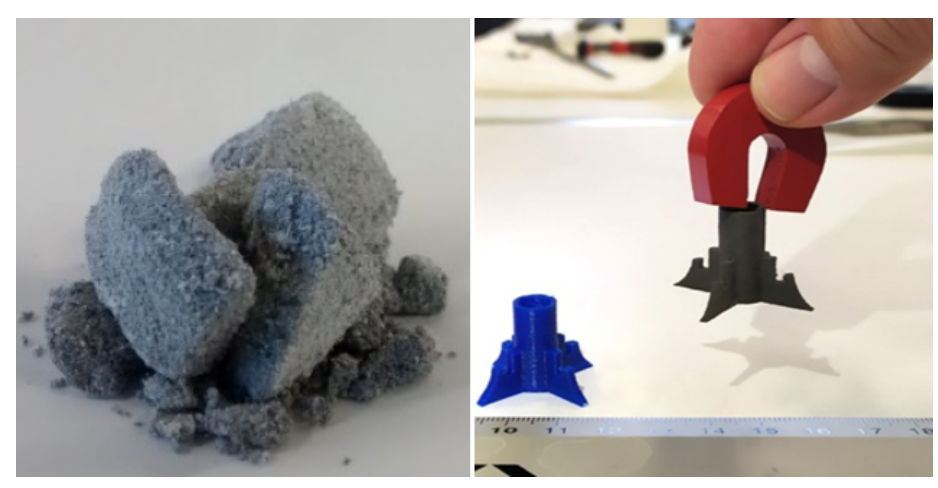

A processed powder from recycled magnetic material (left) can be used in a 3D printer to create magnets of arbitrary shape (right). Credit: Permanent Magnets and Applications Group/IMDEA Nanoscience from a magnet-producing company in Spain. They then grind the waste material into a powder with submicrometer-sized grains and heat the powder to $1000^{\circ} \mathrm{C}$, where it undergoes calcination, a process that optimizes the microstructure and magnetic properties of the material. Measuring the magnetic properties of this processed powder, the team finds that it has superior properties to the original residue with a 3.5-fold increase in its coercivity (a measure of the resistance of a magnetic material to changes in its magnetization) and a 25\% increase in its remanence (residual magnetization).

This powder can then be returned to the company for reprocessing into common ferrite magnets. Casaleiz and his colleagues have also shown that it can be used to create a ferrite-polymer composite for 3D printing of magnets, something that hasn't been done using recycled materials before. 3D printing has advantages over traditional magnet-making techniques, as the magnets can be made at lower temperatures, reducing energy costs, and they can be printed into a significantly wider array of shapes.

For 3D printing, the team mixes the powder with a polymer called acrylonitrile butadiene styrene and then extrudes the mixture into long filaments. These filaments are fed into a 3D printer, where they are heated slightly before being deposited on a surface. Using this method, the team has printed a variety of objects, from hexagons and rings to intricately patterned cylinders, all of which retain the magnetic properties of the original powder.

Casaleiz says that the recycled 3D-printed magnets that he and his colleagues have made could find use in technologies ranging from electric vehicles to home appliances. He also 
thinks that these ferrite-based magnets could help replace rare-earth magnets, such as those made from neodymium, whose mining can adversely impact the environment.

Ferrite-based magnets typically have lower strengths than their rare-earth counterparts, but Casaleiz notes that ferrite magnets have other functional advantages. For example, they are chemically inert, making them resistant to corrosion without any coating. Also, "they are more sustainable, and the materials used to make them are easier to source," he says.

Katherine Wright is the Deputy Editor of Physics. 